\title{
Advantages of Using a Variable Pressure Serial Block Face Scanning Electron Microscope for 3D Volume Analyses.
}

\author{
Claudia S. López ${ }^{1,2}$, Melissa Williams ${ }^{1,2}$ and Cedric Bouchet-Marquis ${ }^{3}$. \\ 1. Multiscale Microscopy Core, Oregon Health \& Science University, Portland Oregon USA. \\ 2. Department of Biomedical Engineering, Oregon Health \& Science University, Portland Oregon USA. \\ 3. Materials \& Structural Analysis, Thermo Fisher Scientific, Hillsboro Oregon USA.
}

Launched by Thermo Fisher Scientific (Formerly FEI Company) in 2014, the Teneo ${ }^{\text {TM }}$ Volume Scope (VS) is a novel Serial Block Face Scanning Electron Microscope (SBF-SEM) that combines mechanical and optical sectioning using FEI's proprietary Multi-Energy Deconvolution (MED) technology. The Teneo VS unravels the complex 3D architecture of cells and tissues in their natural context, which is crucial for gaining an understanding of the structure-function correlation in biological systems. This technology facilitates automated acquisition of large scale 3D cellular and tissue architecture by means of SBF-SEM imaging at isotropic resolution. Resin embedded tissue or cells can therefore be imaged a variable SEM vacuum pressures in a fully automated fashion for reconstruction of large volumes.

For this work, human cardiomyocytes infected with the human pathogen Trypanosoma cruzi, the causing agent of Chagas disease, were analyzed. The infected cells were harvested and washed in $1 \mathrm{X}$ PBS prior to processing. The cell pellet was resuspended in $20 \%$ BSA as cryoprotectant and the sample was processed using High Pressure Freezing (HPF) and Quick freeze substitution (QFS) techniques [1]. During the QFS a cocktail of 4\% OSO4, 0.1\% Uranyl Acetate and 5\% water in acetone was used. After the HPF step the samples were dehydrated in increasing series of acetone:Epon epoxy resin and exchanged three times in $100 \%$ Epon. The samples were flat-embedded between two glass slides using parafilm as spacers and polymerized at $60^{\circ} \mathrm{C}$ for 12 hours. Small pieces of the resulting samples were mounted on a regular blank Epon block or on a carbon-loaded block for easy manipulation as well as screening purposes. Flat embedding techniques are routinely used in any electron microscopy (EM) laboratory and generates a sample with a thickness of less than $0.4 \mathrm{~mm}$. For conventional EM imaging these samples are often remounted using epoxy glue. This process tends to increase chances of building up charges during imaging which could be detrimental to SBF-SEM imaging quality. We have found that mounting flat embedded samples onto a $1 \%$ carbon-loaded resin block, by means of silver epoxy glue, mitigates charging yielding the sample amenable for both SBF-SEM mounting and imaging.

Semi-thin sections $(70 \mathrm{~nm})$ were generated to analyze the sample by Transmission Electron Microscopy (TEM), and for comparison purposes block faces were also imaged by SBF-SEM. The instruments used in this study were: FEI Tecnai Spirit T12 equipped with a bottom mount 16 MB AMT camera and a Teneo VS equipped with a T1 detector for high vacuum imaging and a VS-DBS detector for low vacuum mode imaging. High vacuum mode imaging in the Teneo VS was unsuccessful with these cultured cells due to the charging effect observed in the plain resin surrounding the cells and also within the nucleus (Fig. 1A). Nevertheless, we were able to image this sample under low vacuum (0.5 mBar) without compromising the detection of important features (Fig. 1B). Some residual charging was still observed in the surrounding resin but this issue did not affect the 3D image acquisition. 

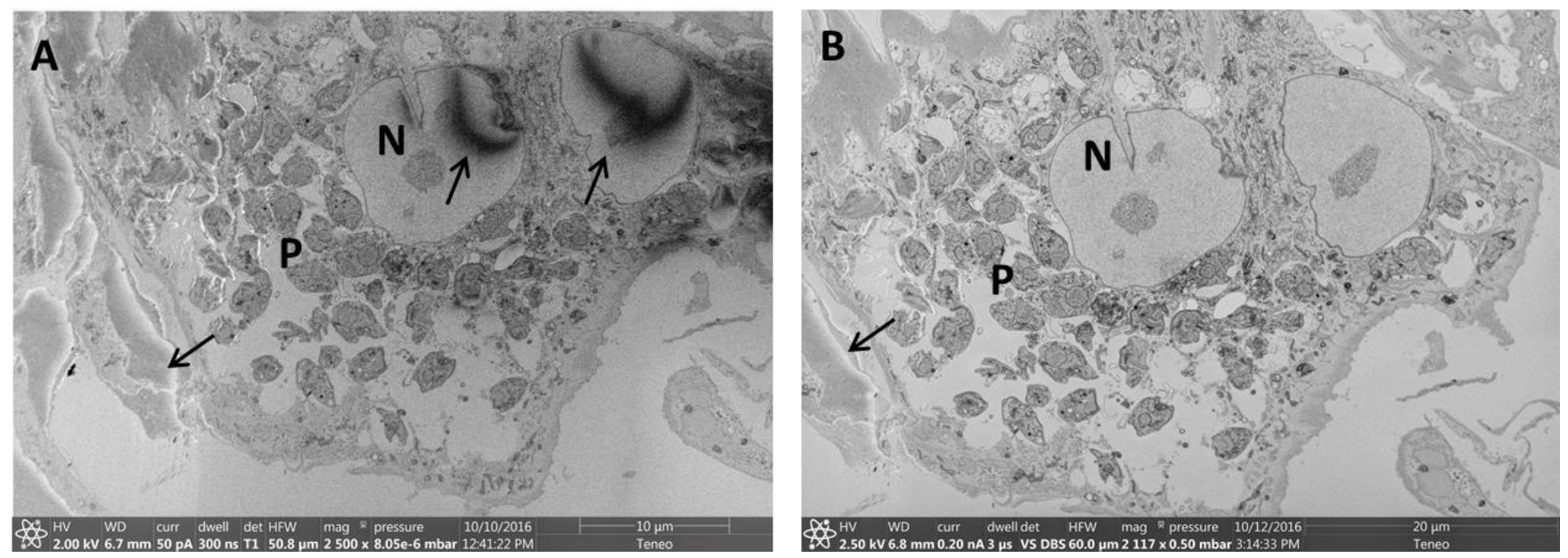

Figure 1. A) Charging defects, indicated by the arrows, are commonly observed in resin embedded cell monolayers making image acquisition difficult. These defects tend to obscure regions of interest interfering with the interpretation of the results. B) Low vacuum acquisition $(0.5 \mathrm{mBar})$ of the same sample shows some charging defects but it is mostly localized in the empty resin and not in the regions of interest. Nucleus (N), entire parasite's cross-sections $(\mathrm{P})$ are labeled.
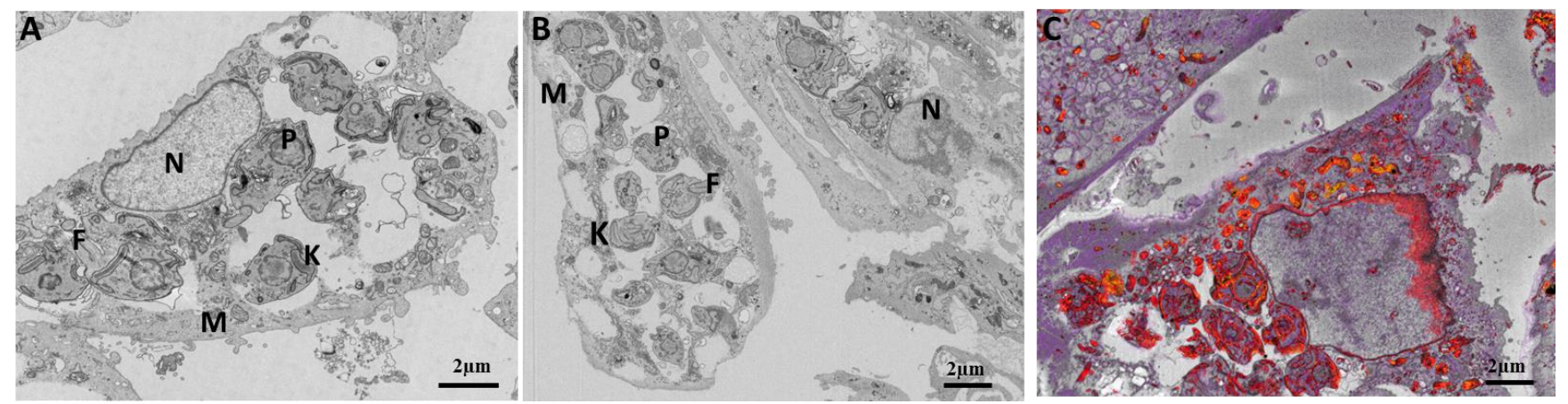

Figure 2. A) TEM image acquired on a FEI Tecnai T12 at 80kV. B) Teneo VS low vacuum image $(0.5$ $\mathrm{mBar}$ ) acquired on a VS-DBS detector at $2.5 \mathrm{kV} 0.2 \mathrm{nA}$. C) The volume rendering shows the limit of the cells as well as many intra cellular parasites.

Figure $2 \mathrm{~A}$ and $2 \mathrm{~B}$ shows comparative images of the same sample acquired on a TEM and the Teneo VS at $0.5 \mathrm{mBar}$. It is clear from these images that several cellular components of the sample such as intra cellular parasites $(\mathrm{P})$, flagella $(\mathrm{F})$, kinetochores $(\mathrm{K})$, cellular mitochondria $(\mathrm{M})$ and nuclear membranes are identified using both techniques. Our results indicate that the low vacuum acquisition does not compromise the resolution of the images. Even though the images acquired in low vacuum mode show in general more noise as compared to the high vacuum ones, it is easily removed from the images by applying a simple 2D or 3D Gaussian filter [2] [3].

\section{References:}

[1] K.L. McDonald \& R.I. Webb. Journal of Microscopy, 243, Pt 3 (2011), p. 227.

[2] We thank Dr. Marie Pierre Hasne for providing the samples used in this study.

[3] Electron microscopy was performed at the Multiscale Microscopy Core (MMC) with technical support from the OHSU-FEI Living Lab Collaboration and the OHSU Center for Spatial Systems Biomedicine (OCSSB). This project was also supported by a Pilot Project Grant from the OCSSB to CSL. 